\title{
On the development and parameter identification of Schapery-type constitutive theories
}

\author{
Martin Lévesque • Katell Derrien • Didier Baptiste • \\ Michael D. Gilchrist
}

Published online: 10 June 2008

(C) Springer Science+Business Media, B. V. 2008

\section{Erratum to: Mech Time-Depend Mater \\ DOI 10.1007/s11043-008-9052-y}

Below equations (26) it is written that:

"where $p_{2}, p_{3}$ or $a_{2}, a_{3}>0$ to ensure that $\Psi$ or $G$ are at isolated minimums in the reference state. The same restriction must also apply to $\Psi_{0}$ and $G_{0}$."

In fact, $p_{2}, p_{3}$ or $a_{2}, a_{3}$ do not need to be strictly positive in order to ensure that $\Psi$ or $G$ are at isolated minima in the reference state. Since it is assumed that all the nonlinearizing functions (i.e. the $p_{z}$ or the $a_{z}$ ) are equal to unity in the reference state, $\Psi$ or $G$ are at isolated minima in the reference state, by definition.

As pointed out by Schapery (1969b), the nonlinearizing functions $p_{2}, p_{3}$ or $a_{2}, a_{3}$ can have negative values. For the sake of discussion, consider the constitutive theory obtained in one dimension from (37b) where: a) the elastic part would be negligible; b) all constants are equal to unity and c) only one term in (38b) is used. Since $a_{1}>0$, assume that $a_{1}=1$. Define $\Theta=\frac{\partial a_{3}}{\partial \sigma} \sigma+a_{3}$ (this is the term in front of the integral in (37b)). It is worth

The online version of the original article can be found under http://dx.doi.org/10.1007/s11043-008-9052-y

M. Lévesque $(\varangle)$

CREPEC, Département de Génie Mécanique, École Polytechnique de Montréal, C.P. 6079, succ. Centre-ville, Montréal, Québec H3C 3A7, Canada

e-mail: martin.levesque@polymtl.ca

K. Derrien · D. Baptiste

Laboratoire d'Ingénierie des Matériaux, UMR8006 CNRS, Ecole. Nat. Sup. Arts et Métiers, 151 bd. de l'Hopital, 75013 Paris, France 
considering the shape of constitutive theory (37b) as a function of the signs of $a_{2}, a_{3}$ and $\Theta$ when subjected to a creep load $\sigma_{0}$. Four different expressions for the strain $\varepsilon$ are obtained:

1. If $\left(a_{2}>0, a_{3}>0, \Theta>0\right)$ or $\left(a_{2}>0, a_{3}<0, \Theta<0\right)$, then (37b) becomes

$$
\varepsilon(t)=|\Theta|\left(1-\exp \left[-\left|a_{2}\right| t\right]\right)\left|\frac{a_{3}}{a_{2}}\right| \sigma_{0}
$$

which is consistent with many experimental observations.

2. If $\left(a_{2}>0, a_{3}>0, \Theta<0\right)$ or $\left(a_{2}>0, a_{3}<0, \Theta>0\right)$, then (37b) becomes

$$
\varepsilon(t)=-|\Theta|\left(1-\exp \left[-\left|a_{2}\right| t\right]\right)\left|\frac{a_{3}}{a_{2}}\right| \sigma_{0}
$$

which means that a negative strain is obtained if a positive stress is suddenly applied.

3. If $\left(a_{2}<0, a_{3}>0, \Theta>0\right)$ or $\left(a_{2}<0, a_{3}<0, \Theta<0\right)$, then (37b) becomes

$$
\varepsilon(t)=|\Theta|\left(\exp \left[\left|a_{2}\right| t\right]-1\right)\left|\frac{a_{3}}{a_{2}}\right| \sigma_{0}
$$

which means that the strain varies exponentially with respect to time.

4. If $\left(a_{2}<0, a_{3}>0, \Theta<0\right)$ or $\left(a_{2}<0, a_{3}<0, \Theta>0\right)$, then (37b) becomes

$$
\varepsilon(t)=-|\Theta|\left(\exp \left[\left|a_{2}\right| t\right]-1\right)\left|\frac{a_{3}}{a_{2}}\right| \sigma_{0}
$$

which means that the strain varies exponentially with respect to time and that a suddenly applied positive stress leads to a negative strain.

By examining all the experimental data that we either have generated or found in the literature, and for loadings that led to a viscoelastic behavior for $t_{v}$ time units (see Sect. 4.2, item 1), it seems that only expression (i) above can adequately represent this creep data. The form of expression (iii) could be useful, for example, to represent creep behavior at the onset of failure, which is usually referred to as Phase III creep. Therefore, the restrictions detailed by (53b) are not all required by thermodynamics but are introduced as assumptions in order to represent experimentally observed behavior.

It should also be noted that Gibbs' free energy expansion in (26b) results from some simplifications of the expansion in (16), as initially introduced by Schapery (1969b) (where it is assumed that $A_{r s}$ depends on $\sigma$ ). The expansion in (26b) leads to the same results when using expression (16) with the simplifications introduced by (16) and (37) from reference (Schapery 1997a). Therefore, using the expansions of (26) to derive constitutive theories will lead to a subset of all the constitutive theories that can be obtained using the general arguments introduced by Schapery. Further details can be found either in (Schapery 1969b) or (Schapery 1997a).

\section{References}

Schapery, R.A.: Further Development of a Thermodynamic Constitutive Theory: Stress Formulation. Purdue University (1969b)

Schapery, R.A.: Nonlinear viscoelastic and viscoplastic constitutive equations based on thermodynamics. Mech. Time-Depend. Mater. 1, 209-240 (1997a) 